\title{
Benefit-Risk of Postoperative, Parenteral, Non-Opioid Analgesics Using Multi-Criteria Decision Analysis (MCDA)
}

S. Schug, ${ }^{1,2}$ E. Pogatzki-Zahn, ${ }^{3}$ L.D. Phillips, ${ }^{4}$ M. Noyes Essex, ${ }^{5}$ R. Xia, ${ }^{5}$ A. Reader, ${ }^{6}$ R. Pawinski ${ }^{6}$

${ }^{1}$ Anaesthesiology and Pain Medicine, Medical School, University of Western Australia, Perth, WA, Australia; ${ }^{2}$ Department of Anaesthesia and Pain Medicine, Royal Perth Hospital, Perth, WA Australia; ${ }^{3}$ Department of Anaesthesiology, Intensive Care Medicine and Pain Therapy, University Hospital of Muenster, Muenster, Germany; ${ }^{4}$ Department of Management, London School of Economics and Political Science, London; ${ }^{5}$ ffizer Inc, New York, NY, USA; ${ }^{6}$ Pfizer Ltd, Walton Oaks, Tadworth, UK

\section{INTRODUCTION}

Tools that compare and quantify the benefits and risks of medicines can help to inform and optimise treatment selection.

For postoperative analgesia, this is an issue because multiple treatment options may have different modes of action and different efficacy and safety profiles. ${ }^{1,2}$

Multi-criteria decision analysis (MCDA) can quantify the benefits and risks of individual drugs, both overall and in comparison with each other ${ }^{3-5}$

Here, we used an MCDA model to compare the benefitrisk profiles of different parenteral analgesics in the acute postoperative setting.

\section{METHODS}

An MCDA was constructed to determine and compare the benefit-risk profiles of 8 widely used parenteral, postoperative analgesics (acetaminophen, parecoxib, metamizol, nefopam, ketorolac, tramadol, morphine and diclofenac) and placebo.

Publically available documents were examined to obtain efficacy and safety data for each analgesic and placebo. The benefit-risk profile of each analgesic and placebo was based on 3 favourable criteria (Benefits) and 14 unfavourable criteria (Risks) (Figure 1).

Figure 1. Effects tree for determining the comparative benefit-risk balance of analgesics and placebo

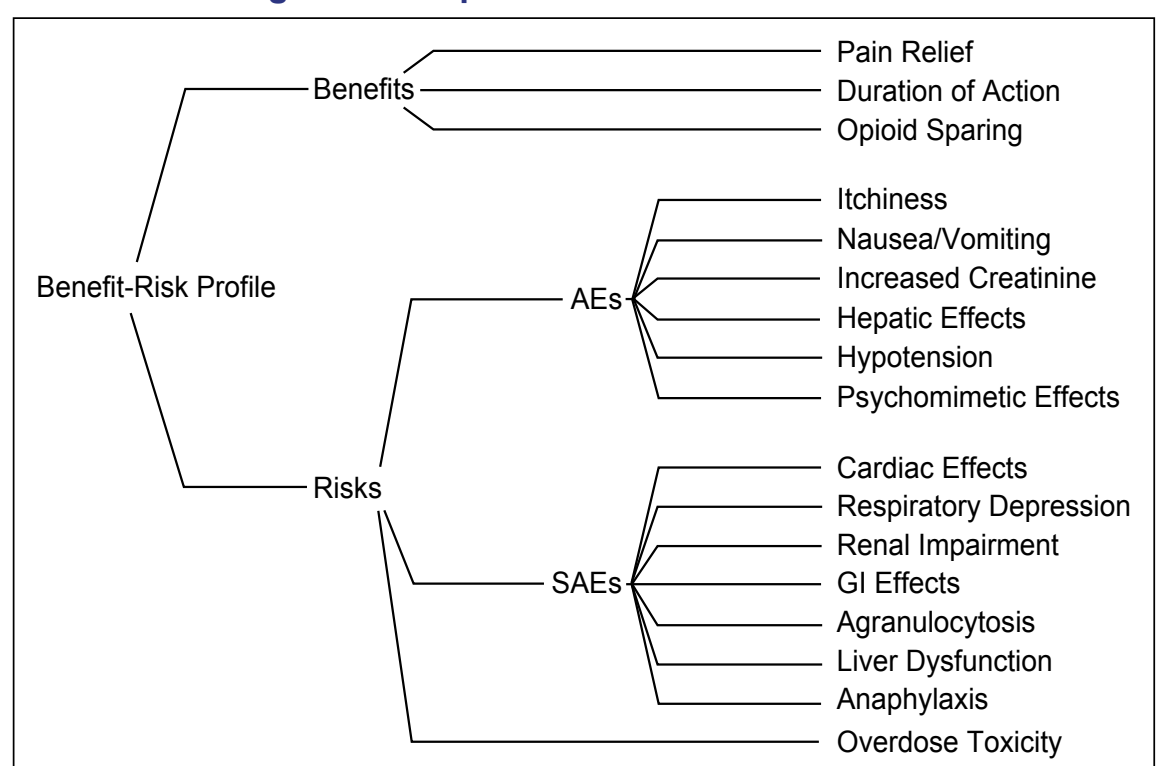

Based on expert clinical opinion, 15 of the 17 criteria were scored from 0-100, with 100 awarded to the drug that scored best and 0 to the drug that scored worst; the remaining drugs were scored according to their standing with each other. The beneficial criteria of Duration of Action and Opioid Sparing were based on available clinical data.

Data for morphine are based on a dose of $4 \mathrm{mg}$.

Swing weights representing the added clinical value from worst to best were assessed as follows:

Benefits (Figure 2A)

Risks, eg, for the serious adverse effects (Figure 2B).

Highest-weighted Benefit against the highest-weighted Risk (Figure 2C).

The weighting process creates a common unit of preference value across all the $0-100$ scales.

Figure 2. Relative weighting of the data

\begin{tabular}{|c|c|c|c|c|}
\hline $\begin{array}{l}\text { A. Pain } \\
\text { Relief }\end{array}$ & Duration & $\begin{array}{l}\text { Opioid } \\
\text { Sparing }\end{array}$ & $\begin{array}{c}\text { C. Pain } \\
\text { Relief }\end{array}$ & $\begin{array}{c}\text { Gl } \\
\text { Effects }\end{array}$ \\
\hline PAR & DIC & PAR & PAR & NEF \\
\hline 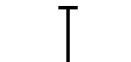 & & 1 & 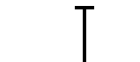 & \\
\hline PBO & PBO & MORPH & $\begin{array}{l}1 \\
\text { PBO }\end{array}$ & $\begin{array}{l}\perp \\
\text { KET }\end{array}$ \\
\hline
\end{tabular}

B. Cardiac Renal Resp GI Agranulo- Liver AnaphEffects Impair Depress Effects cytosis Dysfunc ylaxis

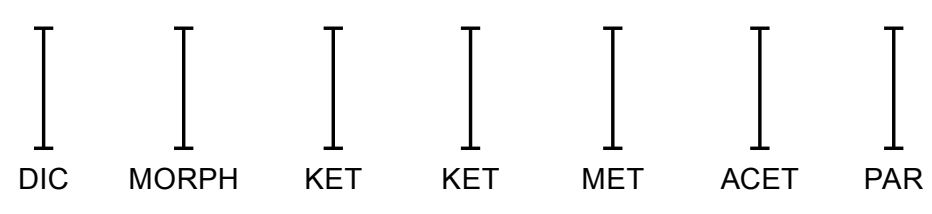

The model was also conducted with a zero weighting placed on the criterion of Opioid Sparing to assess the benefitrisk profile of morphine when Opioid Sparing was not a consideration.

Sensitivity analyses were conducted to assess the impact of varying the weighting of criteria on overall performance. Higher scores for the Risk criteria indicate less risk, ie, higher scores are better.

No statistical comparisons were made between or among analgesics.

\section{RESULTS}

Comparison of the Benefit-Risk Profiles for the 8 Analgesics and Placebo

Overall, Benefits had a relative weighting of $63.6 \%$ and Risks of $36.4 \%$. These two weights are the result of normalising the assessed weights so that they sum to 100 , preserving their original ratios.

Of the Benefits, Pain Relief had the highest weighting (28.9\%)

Of the Risks, Gastrointestinal (GI) Effects had the greatest weighting (11.6\%)

Parecoxib was the most-preferred analgesic (total score = 93), followed by diclofenac (total score $=80$ ) and ketorolac (total score $=75$ ) (Figure 3).

Morphine was the least-preferred option (total score $=57$ ) mainly because it cannot have an opioid sparing effect.

Figure 3. Overall benefit-risk profiles and breakdown by individual benefit and safety criteria

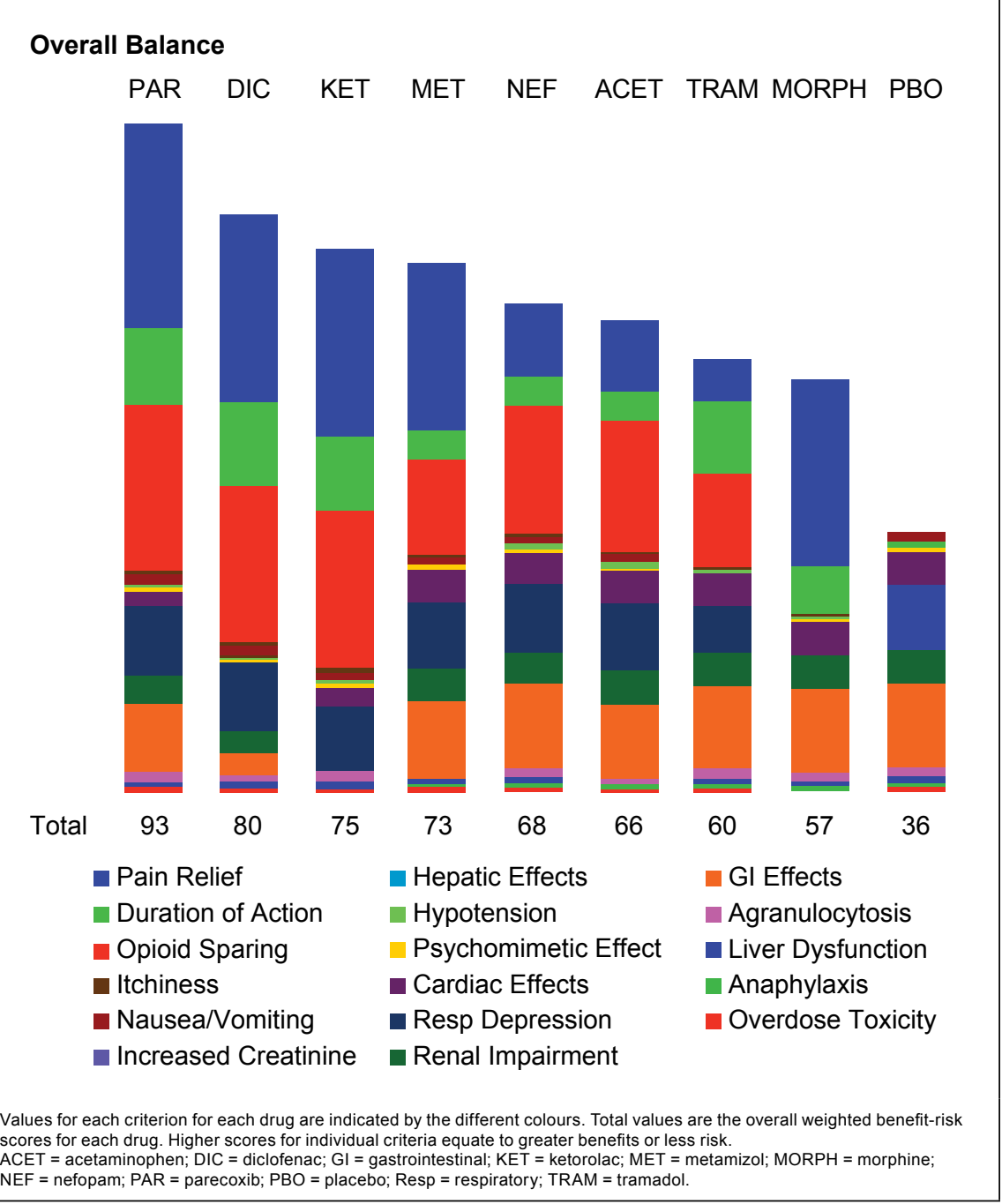

Placing a Zero Weighting on Opioid Sparing Improves the Benefit-Risk Profile of Morphine

When zero weighting was placed on the criterion of Opioid Sparing, parecoxib remained the most-preferred option (total score $=93$ )

However, morphine became the third-most preferred analgesic (total score $=76$ ), with similar score as metamizo (total score $=76$ ) and ketorolac (total score $=74$ ) (Figure 4)

Sensitivity Analysis for Overall Risk Effects

Parecoxib remained the preferred option for a range of weights for the Risks.

Only when the weight for Risk was increased to $>75 \%$ did another analgesic, nefopam, become the most-preferred option.

\section{Sensitivity Analysis for Pain Relief}

Parecoxib remained the preferred option no matter the cumulative weight for Pain Relief.

For a range of cumulative weights from $\sim 10 \%-100 \%$,

diclofenac was the second-most preferred option.
Figure 4. Benefit-Risk profiles when a weighting of zero was placed on the criterion of Opioid Sparing

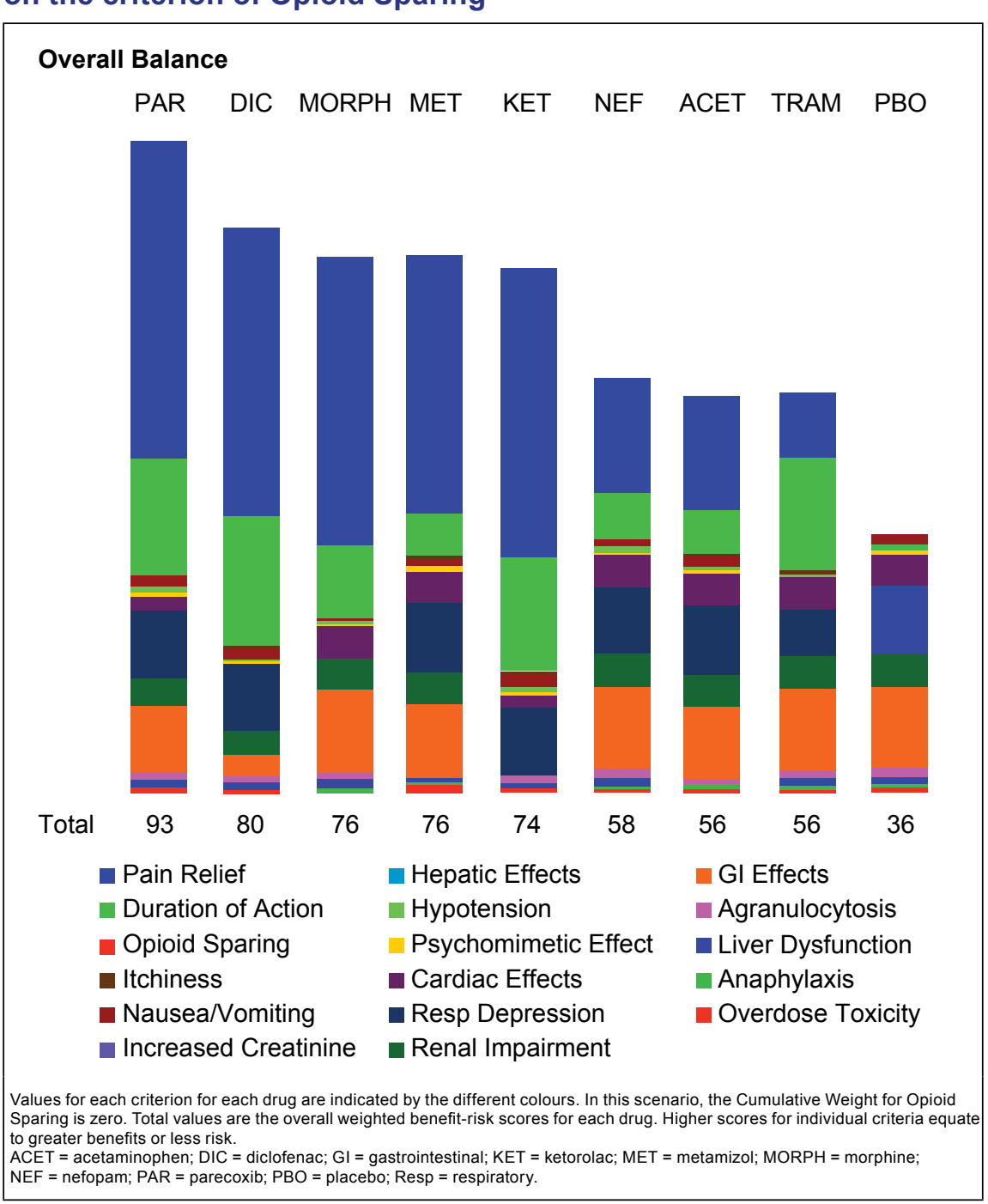

\section{Trade-off Between Benefits and Risk}

Comparison of the analgesics by the separate weighted values for Benefits and Risks, without regard for the tradeoff weight between the Benefits and Risks, is shown in Figure 5.

Parecoxib is close to the ideal (ie, scores of 100 for Benefit and Risk), with no analgesic showing greater benefits. Acetaminophen, metamizol and nefopam each have better values for Risk than parecoxib, but worse values for Benefit. Figure 5. Weighted Benefit preference values vs weighted Risk preference values

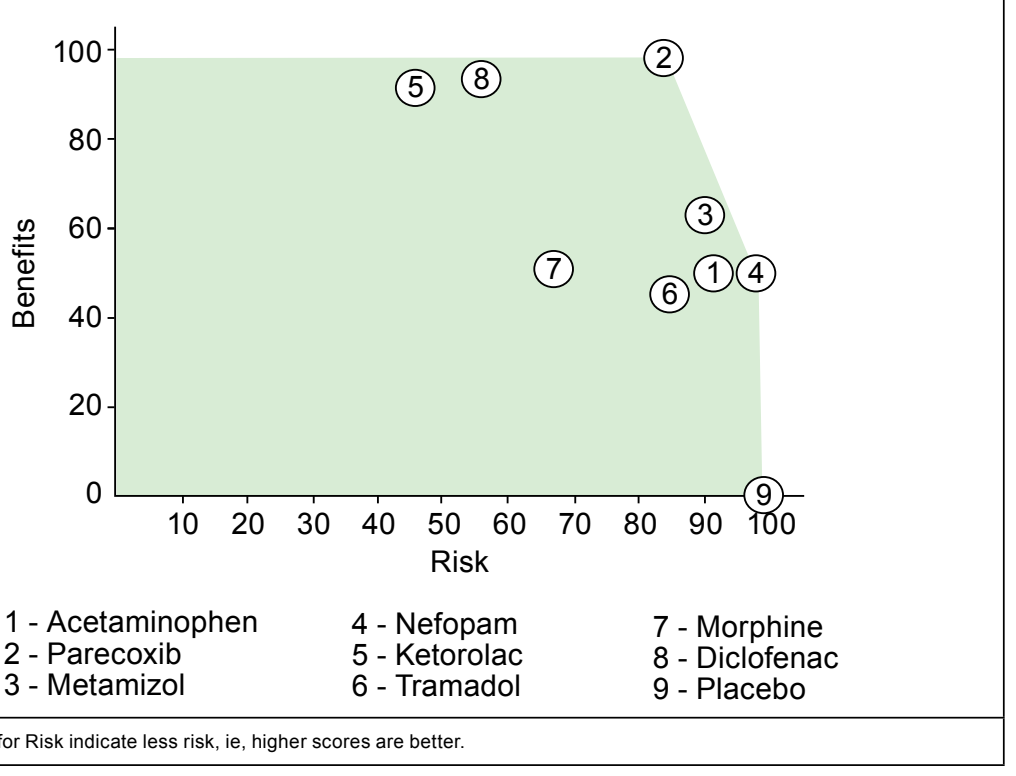

\section{CONCLUSIONS}

Of the 8 parenteral analgesics that were assessed in an MCDA model, parecoxib was the most-preferred option. - High preference scores for Pain Relief, Opioid Sparing, Respiratory Depression and GI Effects drive the high overall score for parecoxib.

- Morphine was the least-preferred analgesic mainly due to its lack of opioid sparing effect. However, when the weighting of Opioid Sparing was placed at zero, morphine became the third-most preferred option.

- This highlights (i) how important Opioid Sparing is when assessing the benefit-risk profile of postoperative analgesics, and (ii) the effectiveness of morphine when Opioid Sparing is not a consideration.

Under a number of sensitivity analyses where the weighting of criteria was varied, eg, for overall Risk and for Pain Relief, parecoxib remained the most-preferred option for the majority of situations.

These results may help to inform clinicians and optimise treatment selection when considering analgesic options in the acute postoperative setting. 